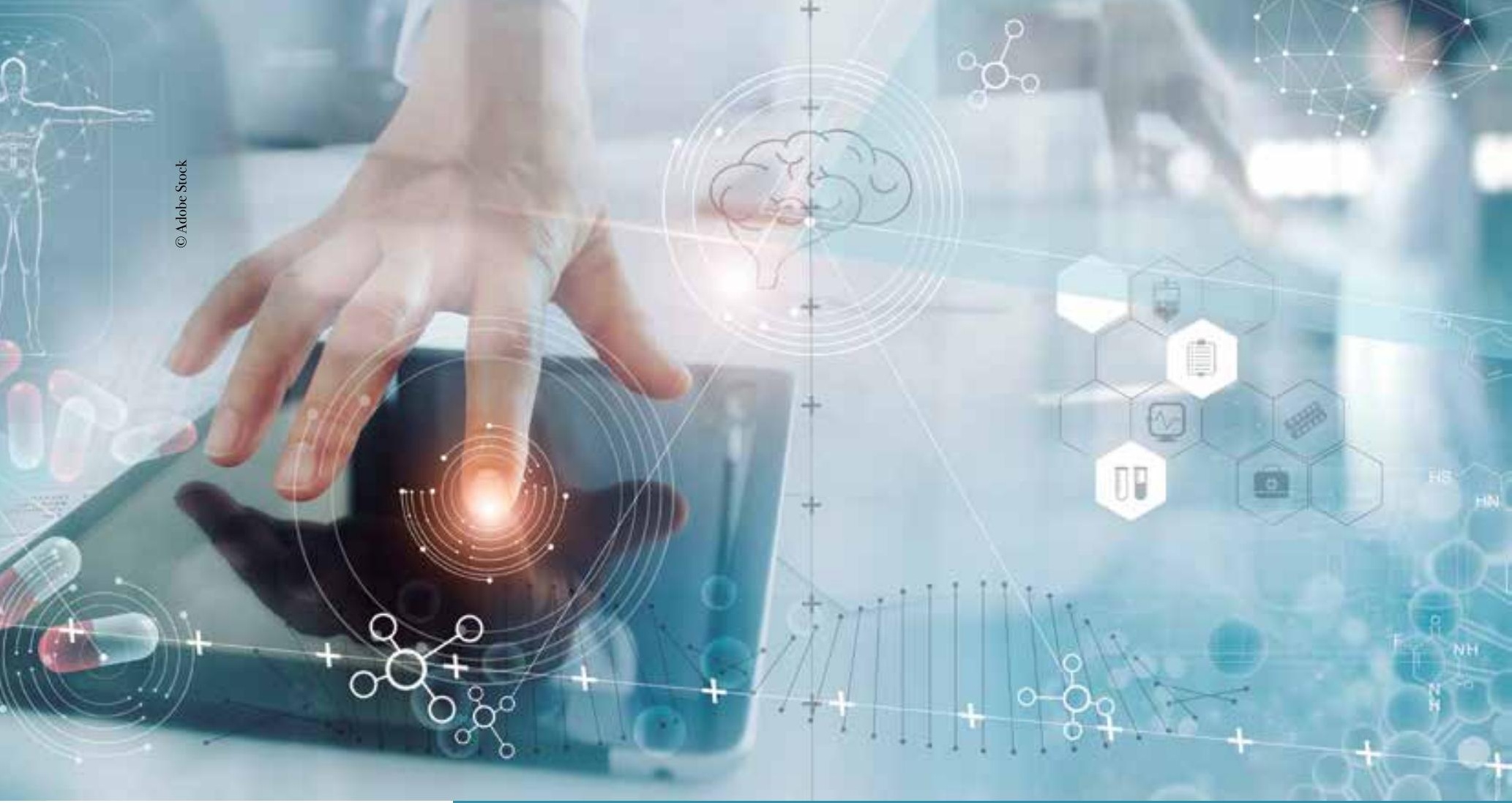

\title{
FERRAMENTAS ONLINE
}

\section{Estímulo à aprendizagem baseada em evidências.}

${ }^{1}$ Bacharelado em Medicina (UFRN).

${ }^{2}$ Graduação em Medicina (UFRN).

3 Graduação em Medicina (UFRN)

4 Graduação em Medicina (UFRN).

${ }^{5}$ Doutorado em Ciências da Saúde (UFRN).

\section{Henrique de Paula Bedaque ${ }^{1}$ Thomas Diniz Papa ${ }^{2}$ Ugor Tomaz Fernandes ${ }^{3}$ Letícia Goes da Silva ${ }^{4}$ \\ Elaine Lira De Medeiros Bezerra ${ }^{5}$}

\section{RESUMO}

Ta era da revolução técnico-científico-informacional, a informação se 1 democratizou de modo nunca antes visto. A internet proporcionou a capilarização do conhecimento até mesmo aos menores aglomerados populacionais, de forma que o aprendizado se tornou mais fluido e dinâmico. Pensando nisso, o grupo do Programa de Educação Tutorial do curso de medicina da UFRN decidiu criar um site para expandir as suas atividades e estimular o estudo da Saúde Baseada em Evidências, através de duas colunas: o PETest e o PETevidências. O PETest é uma coluna de postagem quinzenal de questões comentadas de residência médica de todo o Brasil, ao passo que o PETevidências se compromete em divulgar semanalmente um artigo científico interessante da área médica, de modo a direcionar o estudante de graduação, ou qualquer pessoa interessada no tema, a fazer uso dessa nova ferramenta de aprendizagem. O site já tem 4 anos e é acessado inclusive por estrangeiros, se configurando, portanto, uma excelente atividade de extensão, tanto para os membros do PET quanto àqueles que consomem o conteúdo.

Palavras-chave: Extensão; Universidade; Sociedade; Evidèncias HERRAMIENTAS ONLINE: estímulo al aprendizaje basado en evidencias. 


\section{RESUMEN}

$\mathrm{E}$ la era de la revolución técnico-científico-informacional, la información se democratizó de modo nunca antes visto. La Internet proporcionó la capilarización del conocimiento incluso a los menores aglomerados poblacionales, de modo que el aprendizaje se volvió más fluido y dinámico. En este tema, el grupo del Programa de Educación Tutorial del curso de medicina de la UFRN decidió crear un sitio para expandir sus actividades y estimular el estudio de la Salud Basada en Evidencias, a través de dos columnas: el PETest y el PETevidencias. El PETest es una columna de colocación quincenal de cuestiones comentadas de residencia médica de todo Brasil, mientras que el PETevidencias se compromete a divulgar semanalmente un artículo científico interesante del área médica, para dirigir al estudiante de graduación, oa cualquier persona interesada en el tema, a hacer uso de esta nueva herramienta de aprendizaje. El sitio ya tiene 4 años y es accedido incluso por extranjeros, configurándose, por lo tanto, una excelente actividad de extensión, tanto para los miembros del PET en cuanto a aquellos que consumen el contenido.

\section{Palavras-chave: Extensión; Universidad; La sociedade, Evidencias}

\section{ONLINE TOOLS: stimulation to learn based on evidence.}

\section{ABSTRACT}

I In the era of technical-scientific-informational revolution, the information democratized In a way never seen before. The internet provided the capilarization of the knowledge until the smaller population clusters, so that the learning became more fluid and dynamic. Thinking in that, the group of Tutorial Educarion Program of the UFRN's Medicine course decided to create a site to expand your activities and stimulate the study of the Evidence-Based Health through two columns: the PETest and the PETevidências. The PETest is a column of biweekly posting of commented questions of medical residency from all over the Brazil, while the PETevidências is compromised in a weekly divulgation of an interesting scientific paper in the medical field to direct the graduation student or anybody else interested in the subject to use this new learning tool. The site already has 4 years and it's accessed including by foreigners, setting itself, therefore, an excellent extension's activity both for the PET members as for those who consume the contente.

\section{Palavras-chave: Extension; University; Society; Evidence.}

\section{EM QUE CONSISTE A PRÁTICA A SER RELATADA}

A implementação de um endereço eletrônico com o objetivo de torná-lo um instrumento Ade ensino foi o pilar estimulador para que o Grupo do Programa de Educação Tutorial (PET) do curso de graduação de medicina da UFRN desenvolvesse, em 2OI4, o seu atual site. A decisão por essa forma de comunicação decorreu de suas múltiplas possibilidades de interação e, principalmente, da possibilidade de ultrapassar quaisquer barreiras físicas, dando oportunidade para um ensino mais público e democrático, já que qualquer pessoa que tenha o acesso à rede de computadores com internet pode usufruir das atividades disponíveis (COSTA; FERREIRA, 2OI2).

São duas as principais atividades educacionais que sustentam o site como ferramenta de extensão para o desenvolvimento do conhecimento em saúde: O PETest: se delicie com um desafio; e o PETevidências.

No PETest, elegemos um desafio quinzenal para que o usuário possa testar os seus conhecimentos. A questão é escolhida dentre provas de residência médica de todo o país, permitindo uma discussão acerca do tema envolvido, indo além da simples resolução. Dessa forma, o comentário da questão debate exaustivamente a área do conhecimento que é 
demandada para o êxito, bem como disponibiliza referências bibliográficas atuais e com altos níveis de evidência. Ademais, esse vira um ambiente de reflexão e interatividade ao possibilitar que o internauta deixe sua marca em cada desafio, seja por um like ou mesmo por comentários (COSTA; FERREIRA, 2OI2; JULIANI et al, 2OI2). As perguntas são publicadas quinzenalmente intercaladas com os comentários, para que o usuário tenha estímulo para pesquisar sobre o tema e, após uma semana, possa engrandecer e consolidar o conhecimento ao visualizar o comentário publicado (MEZZARI, 2OII).

O PETevidências, por sua vez, busca trazer para o estudante maior proximidade com a importante e crescente Saúde Baseada em Evidências (SBE), que é o produto do encontro entre os resultados de estudos científicos e as práticas em saúde de profissionais qualificados (SACKETT, 2OO3; LEITE; SÁ, 2OO2). A velocidade a qual artigos são publicados é inédita, no entanto, como estudantes de medicina e futuros profissionais, precisamos estar atentos à qualidade e vieses de tais publicações, o que não é rotineiramente abordado durante a graduação. Nesse contexto, o PETevidências seleciona artigos de grande impacto e utilidade, dentre revistas e jornais de alta relevância científica e nível de recomendação, para realizarmos um resumo crítico sobre os seus resultados, tecendo comentários sobre a metodologia ou sobre sua prática clínica.

Desse modo, o PETevidências possibilita uma maior proximidade do estudante de graduação ao ambiente científico, com evidências de importância e relevância clínica, que podem e devem ser usadas para nortear condutas e decisões terapêuticas e que sejam atrativas à leitura (CASTIEL; PÓVOA, 2002). Assim, é promovido o treinamento para se desenvolver habilidades em análise crítica de artigos.

\section{CONTEXTO EM QUE OCORRE A AÇÃO}

tualmente, a relação do ser humano com a internet, o smartphone e as redes sociais Ajá se tornou integrante e inseparável ao cotidiano, a qual pode se tornar uma ferramenta muito interessante para a educação, por quebrar barreiras e limites inimagináveis há 20 anos. Dessa forma, estamos no momento de adaptação para essa nova relação aluno-aprendizado, no qual, estar em sala com conteúdo passados de forma expositiva,com o objetivo de transformar o sujeito por meio do ensino, já não se mostra eficiente.

É nesse contexto que vemos as ferramentas digitais, inicialmente usadas com fins recreativos, como reais oportunidades de construir uma nova relação aluno-aprendizado. Um site que possa trazer grande acessibilidade, flexibilidade de horário e conhecimento sob demanda são algumas características que atraem o público da graduação ou qualquer outro interessado em aquisição de conhecimento e discussões científicas (SILVA; et al, 20O7). Além disso, certamente, a possibilidade de aprender com interação em rede, por meio de like, compartilhamento e comentários é, sem dúvida, um atrativo maior.

Dessa forma, em 2OI4, o grupo PET - Medicina UFRN percebeu essa oportunidade para colaborar com a educação por meio de uma atividade de extensão que ultrapassasse as barreiras universitárias e pudesse chegar não apenas a estudantes da área da saúde como a qualquer amante da ciência e a interessados no amplo tema da saúde. Assim, nasceu o site do PET, uma ferramenta inovadora de ensino que se apresenta com um grande potencial de disseminação e de utilização de novas metodologias para trazer conhecimento (LEITE; LEITE; MOROSINI, I997).

\section{PARTICIPANTES/INTEGRANTES DA AÇÃO RELATADA}

Programa de Educação Tutorial foi desenvolvido pelo Ministério da Educação (MEC) com o objetivo de trazer estudantes da graduação, orientados por um tutor, para a linha de frente no que tange a transformações pertinentes a ações de ensino, pesquisa e extensão da sua área de atuação. Dessa forma, o PET Medicina UFRN foi criado em 2006 e tem seu foco na transformação do ambiente de aprendizado da área da saúde por meio de atividades que tentem sempre fundir o tripé universitário (ensino, pesquisa e extensão). 
Os I8 membros discentes do PET Medicina UFRN realizam diferentes atividades, entre as quais o Site do PET (petmedufrn.webnode.com) que conta com quatro membros dedicados à sua manutenção e atualização.

\section{METODOLOGIA}

$\mathrm{E}$ m sua construção em 20I4, foi escolhida uma Plataforma de Criação de Site gratuito (Plataforma: Webnode) como forma de tornar o processo de criação mais simples, visto o pouco conhecimento dos membros do PET sobre programação e Webdesign. Assim, por meio das facilidades que essas plataformas de construção proporcionam ao produtor, fomos aprendendo e desenvolvendo habilidades novas que, teoricamente, não fazem parte de uma estrutura curricular de medicina, mas que podem se tornar uma ótima ferramenta pessoal e profissional no futuro (SILVA; CASSIANI; ZEM-MASCARENHAS, 20OI; SILVA; et al, 2007).

Após cerca de 02 meses de familiarização com a plataforma, foram construídos os menus essenciais para mostrar ao usuário a história do PET e, principalmente, informações acerca das atividades desenvolvidas pelo grupo. Entretanto, com a finalização do site "mínimo", percebeu-se que sem a criação de algo interessante para a educação, o site ficaria estático e, assim, teria uma utilidade meramente institucional para mostrar o que é, e o que faz o PET. Foi exatamente nesse momento que nasceu a vontade de ultrapassar as barreiras universitárias, de modo similar a ALVES, para a construção de atividades de ensino, como é hoje o PETest e o PETevidências (ALVES et al, 2006).

Na metodologia do PETest decidimos que a cada duas semanas seria lançada uma nova questão comentada de residência, sendo na primeira semana apenas o desafio e já na segunda, os comentários com as devidas referências bibliográficas utilizadas como embasamento teórico. Além disso, é válido dizer que como forma de não deixar a atividade muito desgastante, tendo em vista a elevada carga horária do curso de medicina, utilizamos as nossas férias para criar um banco de questões já comentadas e, assim, tornar o projeto duradouro. Isto porque, mesmo que os membros do grupo PET estejam sem condições para fazer os comentários num determinado momento, o banco de questões garante segurança à continuidade do projeto. Acreditamos que essa estratégia tem sido fundamental para os quase 3 anos de existência, em periodicidade quinzenal, do PETest.

Ademais, na metodologia do PETevidências decidimos que teríamos uma frequência semanal de postagem em nosso site, com divulgação em outros meios sociais como "instagram" e "Facebook", com um rodízio entre os membros dedicados à manutenção do site para que ninguém ficasse sobrecarregado. Dessa forma, semanalmente temos uma sugestão de artigo científico na área da saúde com seu comentário, utilizando os fundamentos da Saúde Baseada em Evidências e a possibilidade de trazer relevância clínica com base em um conhecimento científico (SACKETT, 2003).

\section{RESULTADOS ALCANÇADOS}

$\mathrm{O}$ atual site do PET Medicina UFRN conta com cerca de I5Oo visitas mensais, sendo os principais motivos: inscrição em outros eventos do PET e participação nas atividades desenvolvidas para o site (PETest e PETevidências). Além disso, com um valor de 6367 visitantes únicos nos últimos dois anos percebemos que para manter essa média de 1500 visitas mensais (com I6.650 páginas visualizadas por mês) temos um público de frequentadores regulares de nosso site, o que infere o destaque para as atividades.

No PETest estamos atualmente com 92 postagens desde sua formação. O que simboliza uma real consolidação da atividade pela sua continuidade e longitudinalidade. Além disso, no último ano iniciamos uma forma mais ativa de divulgação por meio de uso de uma outra rede social: o Instagram. Nele contamos com uma maior praticidade em sua utilização por aparecer de forma simples e rápida no mural de todas as pessoas 
${ }^{1}$ Google Index e o Yahoo Index são métodos quantitativos de avaliação sobre o desempenho da ferramenta de pesquisa em indexar as páginas de um determinado site nas buscas dos usuários, por exemplo, identificando palavras chaves que mais aparecem no site e relacionar com as buscas. que seguem o@petmedufrn. Por fim, contamos com uma média de ro likes por postagem.

Em adição, o PET evidências, que teve seu início em 2oI6, conta com 9I postagens em nosso site e tem como meio divulgador postagens no Facebook, e-mail de turmas e a própria divulgação de pessoas que costumam usar de forma regular esse projeto. Dessa forma, podemos concluir, também, que o projeto está consolidado e terá uma longeva vida buscando sempre aumentar o seu poder de disseminação.

Por fim, é notório que, após 4 anos de sua formação, o site do PET Medicina UFRN se tornou uma grande ferramenta de disseminação de conhecimento acessível a qualquer hora e em qualquer lugar. Até mesmo um reconhecimento do Google sobre o impacto de nosso site ao ser colocado como segundo item no momento da busca "PET Medicina", mesmo havendo diversos sites de outros PETs de medicina pelo Brasil. Além disso, contamos com Google Index ${ }^{\mathrm{I}}$ de 498 e Yahoo Index ${ }^{\mathrm{I}}$ de 987.

\section{Figura I:}

Coluna do PETest

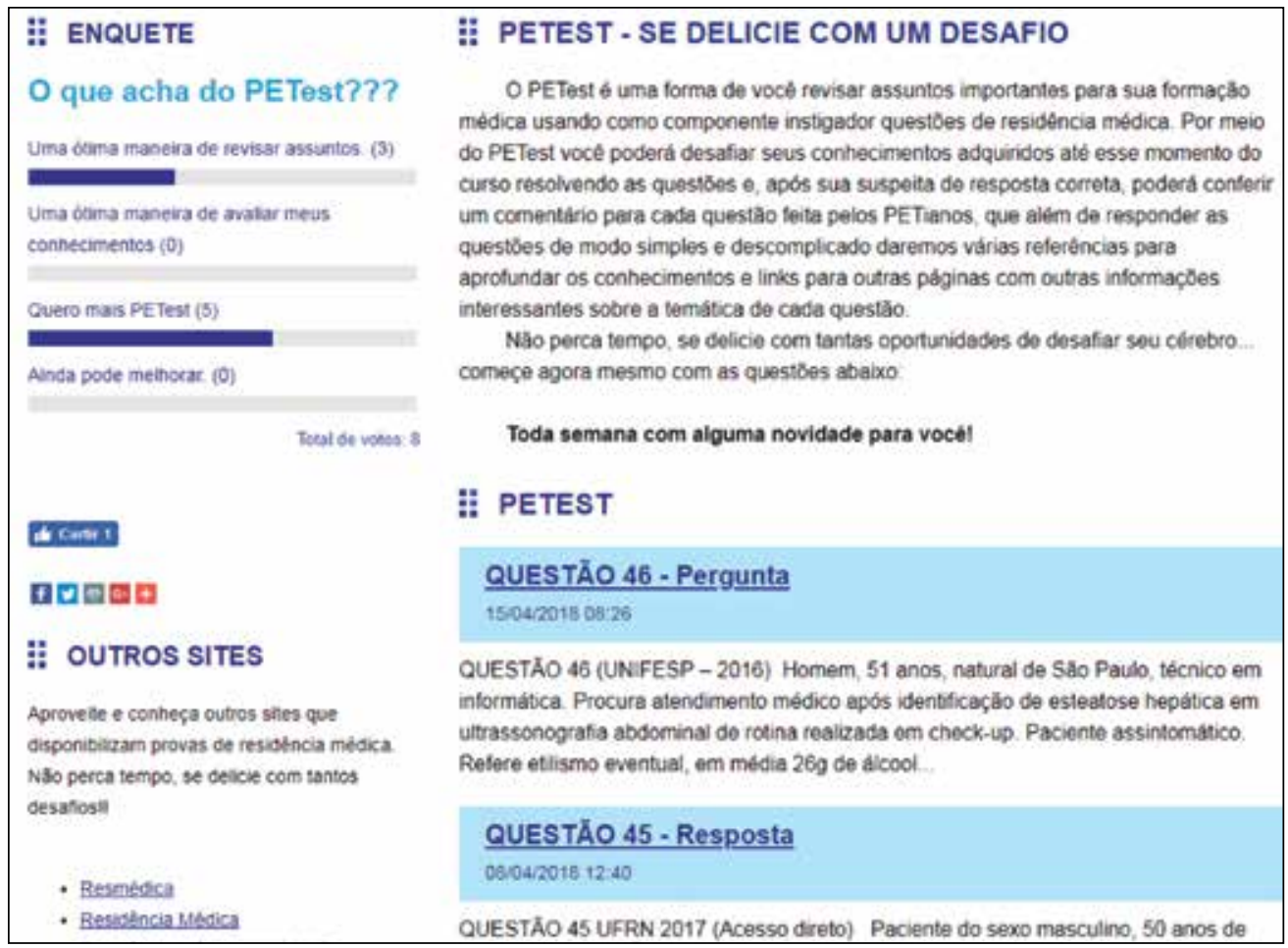

Fonte: Site do PET Medicina UFRN.

\section{O QUE SE APRENDEU COM A EXPERIÊNCIA}

$\mathrm{D}$ iante de todos os desafios encontrados para a construção de um Site por estudantes da graduação (inicialmente leigos em criação na Web) e do desenvolvimento de atividades que pudessem ser atrativas para o público da saúde, acreditamos que nossos maiores aprendizados estão inseridos em três pilares: habilidades em tecnologia; estudo sobre diferentes temas em saúde e conhecimentos sobre Saúde Baseada em Evidências (Epidemiologia, Bioestatística e Medicina Clínica) (SACKETT, 2003).

Nesse contexto, nas habilidades em tecnologias podemos focar mais na parte de criação de site e design pois, apesar das plataformas já oferecerem uma grande simplificação, necessitamos de um estudo prévio para poder tornar todos os nossos objetivos reais. Assim, tivemos que aprender sobre franquia de dados, compactação de arquivos, códigos fonte em HTML e indexação de sites em plataformas de busca. 
Além disso, com o objetivo de deixar o site com uma experiência visual mais agradável, aprendemos a manusear software de manipulação de imagens. Sem dúvidas, esses são conhecimentos que poderão persistir por anos e terão utilidade em um mercado profissional no qual cada conhecimento se torna um destaque.

Ademais, dedicar tempo para a procura de questões de residência e, principalmente, buscar responder tais desafios de uma forma simples, didática e com referências bibliográficas se mostrou muito mais do que um simples trabalho, pois na verdade serviu como uma ferramenta de consolidação de conhecimentos já vistos durante a graduação ou, até mesmo, no aprendizado de novas áreas da saúde. Fica claro perceber quão mais intenso é o aprendizado quando, além de compreender um determinado assunto, é necessário transmitir de forma educativa essa matéria, o que certamente necessita de um maior aprofundamento.

Por fim, sendo a SBE uma área ainda em ascensão e pouco presente na maior parte de nossas graduações, é notável como o estímulo a buscar uma pesquisa interessante, lê-la e criticá-la tem um potencial de fixar conhecimentos, fundamentos, teorias e análises estatísticas (LOPES, 200o). Desse modo, entendemos que a melhor forma de aprender algo é praticando, e isso não é diferente com a SBE (PAOLUCCI, 20O7). É por essa razão que aprendemos tanto lendo os artigos e analisando-os de forma crítica, tendo que escrever de forma simples para que qualquer pessoa possa compreender e se interessar pelo tema.

\section{RELAÇÃO DA PRÁTICA COM OS CONCEITOS DE EXTENSÃO}

$\mathrm{U}$ ma atividade de extensão pode ser interpretada como um ultrapassar das barreiras universitárias para proporcionar uma mudança na vida de pessoas (DE FARIA, 2OOI). Dessa forma, qual seria um ultrapassar de barreiras maior que a tão famosa globalização, responsável por romper com a nossa antiga concepção de limites territoriais e de informação? Assim, construir um site, que pode ser acessado de qualquer lugar e hora, pode ser considerado como umas das ferramentas de maior potencial disseminativo atual (CASTRO, 2004, MENDES, 20IO).

Portanto, quando alunos da graduação de medicina, orientados por uma tutora, e subsidiados pelo Programa de Educação Tutorial desenvolvem mecanismos de educação voltados para qualquer pessoa com interesse na área da saúde, certamente estamos conceituando a prática da extensão (JEZINE, 2004).

\section{REFERÊNCIAS}

ALVES, Vera Lúcia Souza et al. Criação de um web site para enfermeiros sobre pé diabético. Acta Paulista de Enfermagem, 2006.

CASTIEL, Luis David; PÓVOA, Eduardo Conte. Evidence Based Medicine:" a new paradigm for teaching and the provision of care?". Interface-Comunicação, Saúde, Educação, v. 6, n. II, p. II7-I2I, 2002.

CASTRO, Luciana Maria Cerqueira. A universidade, a extensão universitária e a produção de conhecimentos emancipadores. Reunião Anual da ANPED, v. 27, p. I-I6, 2004 .

COSTA, Ana Maria Simões Netto; FERREIRA, Andre Luis Andrejew. Novas possibilidades metodológicas para o ensino-aprendizagem mediados pelas redes sociais Twitter e Facebook. Revista de Ensino de Ciências e Matemática, v. 3, n. 2, p. I36-I47, 2012.

DA SILVA, Jorge Luiz Lima et al. Construção de site na internet sobre saúde da criança e do adolescente: contribuição para processo ensino-aprendizagem. Ciência, Cuidado e Saúde, v. 6, n. 3, p. 363-37I, 2007. 
DE FARIA, Dóris Santos. Construção conceitual da extensão universitária na América Latina. Editora Universidade de Brasília, 200I.

JEZINE, Edineide. As práticas curriculares e a extensão universitária. In: Congresso Brasileiro de Extensão Universitária. 2004. p. I-5.

JULIANI, Douglas Paulesky et al. Utilização das redes sociais na educação: guia para o uso do Facebook em uma instituição de ensino superior. Renote, v. IO, n. 3, $2 \mathrm{OI2}$.

LEITE, Álvaro Jorge Madeiro; SÁ, Marcos. Medicina Baseada em Evidéncias. SANARE-Revista de Políticas Públicas, v. 3, n. I, 2002.

LEITE, Denise; LEITE, D.; MOROSINI, M. Aprendizagens do estudante universitário. Universidade futurante: produção do ensino e inovação. Campinas: Papirus, p. I47-68, 1997.

LOPES, Antonio Alberto da Silva. Medicina Baseada em Evidéncias: a arte de aplicar o conhecimento científico na prática clínica. Rev. Ass. Med. Bras, n. 46(3), p. 285-288, 2000.

MENDES, M. B. et al. Extensão universitária: entenda e estenda a importância dessa idéia. X Jornada de Ensino, Pesquisa e Extensão-JEPEX, 2010.

MEZZARI, Adelina. O uso da Aprendizagem Baseada em Problemas (ABP) como reforşo ao ensino presencial utilizando o ambiente de aprendizagem Moodle. Revista brasileira de educação médica= Brazilian journal of medical education. Vol. 35, n. I (2OII), p. II4-I2I, 2OII.

PAOLUCCI EL DIB, Regina. Como praticar a medicina baseada em evidências. Jornal Vascular Brasileiro, v. 6, n. I, 20O7.

SACKETT, David L. et al. Medicina baseada em evidéncias: prática e ensino. In: Medicina baseada em evidências: prática e ensino. 2003.

SILVA, Flávia B.; CASSIANI, Silvia Helena de Bortoli; ZEM-MASCARENHAS, Silvia H. A Internet e a enfermagem: construção de um site sobre administração de medicamentos. Rev Latino-Am Enferm, v. 9, n. I, p. п6-22, 20OІ. 